\title{
Timing Is Everything: Could Surveillance Imaging Intensity Influence Survival in High-Risk Melanoma?
}

\author{
Madalyn G. Neuwirth, $\mathrm{MD}^{1}$ (D), and Edmund K. Bartlett, $\mathrm{MD}^{1,2}$ \\ ${ }^{1}$ Department of Surgery, Memorial Sloan Kettering Cancer Center, New York; ${ }^{2}$ Memorial Sloan Kettering Cancer Center, \\ New York, NY
}

Ibrahim et al. ${ }^{1}$ present a single-center retrospective study of high-risk melanoma patients after surgical resection and describe the association between surveillance imaging and patient outcomes. The utility of surveillance imaging for melanoma is remarkably understudied and has become increasingly interesting in this era of effective systemic therapy, with some data suggesting that treatment is more effective with a lower disease burden ${ }^{2,3}$.

The authors distinguish between patients whose recurrence is detected radiologically as asymptomatic surveillance-detected recurrence (ASDR) and those who present with clinical, or symptomatic, recurrence (SR). Importantly, the authors found that patients with ASDR had shorter median follow-up periods and were more likely to undergo subsequent systemic therapy than those with SR at presentation. Furthermore, patients with ASDR had better overall survival than those with SR (median, 39.2 vs 23.2 months; $p=0.02$ ), and this difference persisted after multivariate adjustment for numerous prognostic factors. This finding suggests that ASDR may be an important prognostic factor at the time of recurrence that can be incorporated into the counseling of patients and future riskmodeling studies.

In response to Ibrahim et al. Impact of Imaging Intensity and Survival Outcomes in High-Risk Resected Melanoma Treated by Systemic Therapy at Recurrence. ASO-2019-10-2465.R1.

\section{(C) Society of Surgical Oncology 2020}

First Received: 3 April 2020;

Published Online: 28 May 2020

E. K. Bartlett, MD

e-mail: bartlete@mskcc.org
No clear consensus exists on which imaging methods should be used for surveillance. Most of the patients in this study underwent axial imaging in the form of computed tomography (CT) or positron emission tomography (PET)/ $\mathrm{CT}$, without significant differences between the ASDR and SR groups.

Outside the scope of this study, but critical in the decision to image patients, is the cost of the imaging ${ }^{4,5}$. This cost is not solely financial, but additionally includes false-positives with subsequent tests/biopsies, patient anxiety, and radiation exposure. It must be recognized that these harms are incurred by all patients, not only by the subset that experiences recurrence ${ }^{6}$. Thus, the authors' conclusion calling for an "optimal risk-adapted imaging frequency" is a thoughtful approach to ensure that the threshold for imaging is appropriate to the number of anticipated events.

Finally, Ibrahim et al. ${ }^{1}$ demonstrate an association between imaging intensity and ASRD as well as between ASRD and disease outcome for treated patients. This finding raises the possibility of a causal relationship between more intensive imaging and oncologic outcome, presumably resulting from greater efficaciousness of earlier treatment. As the authors appropriately acknowledge, a randomized study is required to answer this, but the current study provides some insights into this interesting hypothesis.

As expected, the ASDR patients were less likely to have brain metastases and had a lower tumor burden than those with SR. Perhaps surprisingly, time to recurrence did not differ significantly between the asymptomatic and symptomatic patients, which the authors suggest argues against lead time bias. Although the reasons for this finding are 
likely multifactorial, the authors appropriately addressed lead time bias by measuring survival from initiation of surveillance.

Instead of attributing a survival benefit to more intensive imaging, it might be more plausible to conclude that ASDR and SR recurrences represent biologically different diseases. Indeed, the authors demonstrated this biologic difference by finding that ASDR was associated with significantly longer survival than SR even for untreated patients (Fig. S5C). Intriguingly, for the treated patients stratified by ASDR versus SR, despite the markedly different median survivals, the plateaus of the survival curves seemed to converge (Fig. S5A). This is a very limited sample size, but if true would suggest that ASDR is associated with more indolent disease, and that ultimately the long-term survivors are those whose disease course was altered by effective therapy. Longer follow-up evaluation of this population is needed for any definitive conclusions.

Certain limitations to the current study warrant discussion. First, the surveillance intervals and methods of imaging during those intervals were highly variable. Across both recurrence groups, the follow-up intervals ranged from 0 to 12 months, with an average imaging interval of 7.7 months for the stage 2 patients and 5.7 months for the stage 3 patients. Most of the patients underwent axial imaging (CT or PET/CT), with only $9 \%$ of the total cohort undergoing a combination of imaging methods. With such variation, except for the trend of shorter surveillance periods identified for the ASDR patients, it was not feasible to determine an "ideal" period to guide clinical practice. Furthermore, dual-imaging methods (i.e., CT scan and nodal basin ultrasound) may increase the sensitivity for detecting both regional and distant recurrence, consequently increasing the proportion of ASDR patients. Absent is discussion of brain MRI, a modality for which detection of ASDR has significant potential to impact on disease course.

Second, although systemic therapy (immune therapy, targeted therapy, or chemotherapy) was included in the subset analyses as a salvage method at the time of recurrence, effective adjuvant therapy for high-risk melanoma patients had not yet emerged during the period of this study. Although the patients with ASDR were more likely to receive systemic treatment for their recurrence, it appears that no patients from either group received effective adjuvant therapy before their recurrence. As such, the applicability of the findings to the adjuvant therapy population are unknown. Early detection after adjuvant therapy may have a different prognostic significance because by definition such a recurrence is treatment resistant.

Overall, Ibrahim et al. ${ }^{1}$ emphasize an important aspect of melanoma management, namely, that surveillance imaging is understudied among high-risk patients. The current study effectively demonstrated the significant prognostic value of ASRD versus SR and raises for further study the potential of imaging intensity to influence oncologic outcomes. Currently, it would be prudent for clinicians to establish and apply consistent imaging surveillance protocols that allow assessment of the impact that imaging will have in the future.

\section{REFERENCES}

1. Ibrahim A, Le May M, Bosse D, et al. Imaging intensity and survival outcomes in high-risk resected melanoma treated by systemic therapy at recurrence. Ann Surg Oncol. https://doi.org/10. 1245/s10434-020-08407-8.

2. Pires da Silva I, Lo S, Quek C, et al. Site-specific response patterns, pseudoprogression, and acquired resistance in patients with melanoma treated with ipilimumab combined with anti-PD-1 therapy. Cancer. 2020;126:86-97.

3. Warner AB, Postow MA. Bigger is not always better: tumor size and prognosis in advanced melanoma. Clin Cancer Res. 2018;24:4915-7.

4. Pace LE, Keating NL. A systematic assessment of benefits and risks to guide breast cancer screening decisions. JAMA. 2014;311:1327-35.

5. Reyes C, DaCosta Byfield S, Linke R, Satram-Hoang S, Teitelbaum AH. The burden of metastatic melanoma: treatment patterns, healthcare use (utilization), and costs. Melanoma Res. 2013;23:159-66.

6. Nijhuis AAG, Dieng M, Khanna N, et al. False-positive results and incidental findings with annual CT or PET/CT surveillance in asymptomatic patients with resected stage III melanoma. Ann Surg Oncol. 2019;26:1860-8.

Publisher's Note Springer Nature remains neutral with regard to jurisdictional claims in published maps and institutional affiliations. 